\section{AB013. CXCL4-DNA immune complexes drive inflammation in systemic sclerosis by amplifying TLR9-mediated interferon- $\alpha$ production}

\author{
Ernest Y. Lee ${ }^{1,2,3}$, Roberto Lande ${ }^{4}$, Carlo Chizzolini ${ }^{5}$, \\ Gerard C. L. Wong ${ }^{1}$, Loredana Frasca ${ }^{4}$ \\ ${ }^{1}$ Department of Bioengineering, ${ }^{2}$ Division of Dermatology, UCLA, \\ Los Angeles, CA, USA; ${ }^{3}$ UCLA-Caltech Medical Scientist Training \\ Program, David Geffen School of Medicine, UCLA, Los Angeles, CA, \\ USA; ${ }^{4}$ National Center for Drug Research and Evaluation, Istituto \\ Superiore di Sanità, Rome, Italy; ${ }^{5}$ Immunology \&amp, Allergy and \\ Immunology \&amp, Pathology, University Hospital and School of \\ Medicine, Geneva, Switzerland \\ Correspondence to: Roberto Lande; Loredana Frasca. National Center \\ for Drug Research and Evaluation, Istituto Superiore di Sanità (ISS), \\ viale Regina Elena 299, 00161 Rome, Italy. Email: roberto.lande@iss.it; \\ loredana.frasca@iss.it; Gerard C. L. Wong. UCLA Bioengineering, \\ 420 Westwood Plaza, 5121 Engineering V, UCLA, Los Angeles, CA \\ 90095-1600, USA. Email: gclwong@seas.ucla.edu.
}

\begin{abstract}
Systemic sclerosis (SSc) is a chronic autoimmune disease characterized by inflammation-driven tissue fibrosis and vasculopathy. CXCL4 is a chemokine overexpressed in SSc and represents an early serum biomarker of severe disease. CXCL4 likely contributes to inflammation via chemokine signaling pathways, but the exact role of CXCL4 in SSc pathogenesis is unclear. Here, we combine $\mathrm{X}$-ray scattering, confocal microscopy, immune cell experiments, and analysis of SSc skin biopsies to elucidate an unanticipated mechanism for CXCL4-mediated immune amplification in SSc. We find that CXCL4 organizes "self" and microbial DNA into liquid crystalline immune
\end{abstract}

complexes that drastically amplify Toll-like receptor 9 (TLR9)-mediated plasmacytoid dendritic cell (pDC) hyperactivation and interferon- $\alpha$ production. Surprisingly, this immunomodulatory activity is independent of CXCR3, the CXCL4 receptor. Importantly, we find that CXCL4DNA complexes are present in vivo and correlate with type I interferon (IFN-I) levels in SSc blood, and that CXCL4-positive skin pDCs coexpress IFN-I-related genes. Taken together, we establish a direct link between CXCL4 overexpression and the IFN-I-gene signature in SSc and identify a potential therapeutic opportunity to disrupt inflammation in SSc by inhibiting the self-assembly of CXCL4-DNA complexes. Our findings are consistent with an emerging general paradigm in which nucleic acidscaffolding proteins like antimicrobial peptides, bacterial amyloids, and chemokines can drive autoimmunity by modulating innate immune receptors without being direct agonists.

Keywords: Systemic sclerosis (SSc); scleroderma; CXCL4; Toll-like receptor 9 (TLR9); self-assembly; immune complexes; nanocrystals

doi: 10.21037/atm.2021.AB013

Open Access Statement: This is an Open Access article distributed in accordance with the Creative Commons Attribution-NonCommercial-NoDerivs 4.0 International License (CC BY-NC-ND 4.0), which permits the noncommercial replication and distribution of the article with the strict proviso that no changes or edits are made and the original work is properly cited (including links to both the formal publication through the relevant DOI and the license). See: https://creativecommons.org/licenses/by-nc-nd/4.0/.

Cite this abstract as: Lee EY, Lande R, Chizzolini C, Wong GCL, Frasca L. CXCL4-DNA immune complexes drive inflammation in systemic sclerosis by amplifying TLR9-mediated interferon- $\alpha$ production. Ann Transl Med 2021;9(5):AB013. doi: 10.21037/atm.2021.AB013 